\title{
Sentinel lymph node biopsy and neoadjuvant chemotherapy in the management of early breast cancer: Safety considerations and timing
}

\author{
J Edge, ${ }^{1}$ MMed (Surg); S Nietz, ${ }^{2}$ FCS (SA) \\ ${ }^{1}$ Christiaan Barnard Memorial Hospital, Cape Town, South Africa \\ ${ }^{2}$ Department of Surgery, Charlotte Maxeke Johannesburg Academic Hospital and University of the Witwatersrand, Johannesburg, South Africa
}

Corresponding author: J Edge (dr@jennyedge.co.za)

Over the last decades, breast cancer treatment has become more personalised. Treatment plans are based on the biology of the tumour rather than the stage. Consequently, neoadjuvant chemotherapy (NACT) is commonly the primary therapy for early breast cancer as well as locally advanced disease. Sentinel lymph node biopsy (SLNB) is standard axillary management for women with node-negative disease. This review looks at the relevant literature and gives guidance on the timing of SLNB when NACT is planned and evaluates the safety of performing an SLNB rather than an axillary clearance.

S Afr Med J 2017;107(6):497-500. DOI:10.7196/SAMJ.2017.v107i6.12239

Traditionally, operable breast cancer has been treated by primary surgery followed by adjuvant chemotherapy, radiotherapy, endocrine and targeted therapy as indicated. Primary systemic chemotherapy, more commonly known as neoadjuvant chemotherapy (NACT), was reserved for large inoperable tumours or for inflammatory breast cancer. Our current decisions on local therapy remain based on historical data, where surgery was the primary treatment modality.

The extent of surgery in the treatment of breast cancer has dramatically decreased following a surgical paradigm shift from maximum tolerated therapy towards minimum required therapy ${ }^{[1]}$ These shifts include the transition from routine axillary lymph node dissection (ALND) to sentinel lymph node biopsy (SLNB) in node-negative patients, and the transition from routine mastectomy to breast-conserving therapy. Conversely, the use of NACT in early breast cancer has increased dramatically over the past decade. This trend poses new challenges in the management of patients with early breast cancer, specifically with regard to decision-making on the management of the axilla and adjuvant radiation.

Although endocrine therapy has a well-established role in the neoadjuvant setting, this review is confined to the use of NACT.

$\mathrm{NACT}$ in early breast cancer has no proven survival benefit, ${ }^{[2]}$ but has other potential advantages:

- It allows assessment of the success of systemic therapy by monitoring the clinical and radiological responses.

- Tumour size and node involvement are reduced. ${ }^{[3]}$

- Breast conservation rates increase by $10-30 \%{ }^{[3-6]}$

- A pathological complete response (pCR) is a significant prognostic factor. ${ }^{[7]}$

- A large proportion of patients in Europe and the USA are enrolled in clinical trials, which allows an evaluation of response to therapy.

- It allows more axilla-preserving surgery.

The use of systemic therapy as the primary treatment modality also fits into the modern perception of breast cancer as a systemic disease. ${ }^{[8,9]}$ It is well recognised that tumour behaviour is different in each patient and tumour biology has superseded the traditional approach of anatomical staging in treatment decisions. Furthermore, the primary tumour has a different biological behaviour compared with the more indolent lymph nodes. Lymph nodes are better thought of as being 'indicators but not governors of survival. ${ }^{[10]}$

\section{Response of axillary nodes to NACT}

Nodal response to NACT is an important prognostic marker and patients who achieve a pCR have improved overall survival and disease-free survival. ${ }^{[7,11]}$ Several studies have evaluated the response of positive axillary nodes to NACT (Table 1).

Response rates vary according to tumour biology. Hormone receptor-positive patients are less likely to achieve pCR than those with triple-negative or human epidermal growth factor receptor 2 (HER2)-positive breast cancer, highlighting the importance of patient selection. ${ }^{[15,16]}$ With the addition of targeted therapy in HER2-positive patients, the conversion rates have increased to $>70 \% .{ }^{[18]}$

Besides the prognostic implications of evaluating response, the use of NACT has the attractive potential to increase axilla-preserving surgical therapy by reducing the need for ALND in patients who are node-negative after NACT.

Although removal of the lymph nodes may have little impact on survival, it adds to local control. In early breast cancer, lymph node status remains one of the most important prognostic factors and it is imperative to accurately stage all patients prior to starting any

Table 1. Summation of principal studies on nodal response to NACT

\begin{tabular}{|c|c|}
\hline Study & Nodal pCR, $n(\%)$ \\
\hline Koolen et al ${ }^{[12]}$ & $80(40.0)$ \\
\hline Park et al..$^{[13]}$ & $178(41.0)$ \\
\hline Hieken et al. ${ }^{[14]}$ & $272(38.5)$ \\
\hline Boughey et al..$^{[1]}$ & $649(41.0)$ \\
\hline Mamtani et al. ${ }^{[16]}$ & $195(49.0)$ \\
\hline Alvarado et al. ${ }^{[17]}$ & $150(42.0)$ \\
\hline
\end{tabular}


therapy, including NACT. Therefore, all patients require a clinical and sonographic assessment of the axilla.

After assessment, patients fall into two groups:

- Clinically node-negative (cN0) patients, with no sign of regional metastases on clinical examination and imaging.

- Clinically node-positive (N1) patients, with signs of regional metastases on clinical examination and/or imaging, which must be proven by fine needle aspiration (FNA) or core biopsy of the nodes.

The first group all require SLNB (ALND for node-negative patients has become obsolete in contemporary breast cancer surgery). The considerations are:

- the timing of the procedure - should it be performed before or after NACT?

- whether a patient who had a positive SLNB prior to NACT could have a repeat SLNB after NACT to increase the rate of axillary preservation?

The second group of patients may continue to be node-positive after NACT or may convert to node-negative. Patients who present with nodal involvement and have persistent nodal involvement after NACT should all have ALND; there is no role for SLNB. However, if patients convert to a clinically node-negative state, can one safely perform an SLNB and preserve the axilla?

\section{Clinically node-negative patients prior to NACT \\ Accuracy of SLNB after NACT}

Data on accuracy and safety of SLNB were based on patients who had surgery first. Therefore, there were initial major concerns about the false-negative rate (FNR) and the feasibility and reliability of SLNB after NACT. However, Hunt et al. ${ }^{[19]}$ compared the accuracy of SLNB in 575 patients after NACT with 3746 patients undergoing primary SLNB, which showed comparable results. The safety of the procedure after NACT was confirmed by various other studies and two metaanalyses. ${ }^{[20,21]}$ The National Comprehensive Cancer Network (NCCN) guidelines allow SLNB both before and after NACT. ${ }^{[2]}$

\section{Timing of SLNB}

\section{SLNB before NACT - the 'up-front' procedure}

SLNB prior to NACT reflects the 'true' stage of the patient and allows accurate prognostication. Proponents for the up-front procedure have argued that a positive node may indicate the use of NACT; in the authors' experience this is uncommon and the indication for NACT is made most often by consideration of tumour biology. The ratio of the primary lesion to the breast size may also be an indication for primary systemic therapy. In some cases, though, a single positive node will indicate the use of radiotherapy after a mastectomy. This can be extremely important information in the planning of reconstructive approaches.

If the sentinel node is free of disease, no further surgical management of the axilla is required. If the node is involved, should the patient have an ALND at the time of definitive surgery or a second SLNB? This question was answered by the SENTinel NeoAdjuvant (SENTINA) trial. ${ }^{[23]}$ Clinically node-negative patients with a positive SLNB prior to NACT underwent a second SLNB after chemotherapy. The identification rate (IR) was $61.0 \%$ and the FNR $51.6 \%$. The trial showed clearly that a second SLNB after NACT is not plausible. From a clinical perspective this is important; when we decide on an up-front SLNB, we deny more patients the opportunity of NACT converting them to node-negative status and preserving the axilla. When the SLNB is positive, we are committed to an ALND. In general, a patient who has an up-front SLNB will always require a second operation, regardless of nodal status.

\section{SLNB after NACT}

This approach allows a greater rate of minimal axillary surgery, given that many patients will convert to node-negative (Table 2). ${ }^{[19]}$

If the sentinel node is negative, no further surgical management is required and the patient is spared the morbidity of an ALND. Furthermore, the patient is spared a second operation with an up-front SLNB. However, a negative SLNB after NACT can create uncertainty regarding the need of adjuvant radiation.

If the nodes are found to be involved, the patient should undergo ALND.

Table 2. Identification rate, false-negative rate and local regional recurrence rate following SLNB before and after $\mathrm{NACT}^{[19]}$

\begin{tabular}{lll}
\hline & $\begin{array}{l}\text { Primary SLNB, \% } \\
(\boldsymbol{n}=\mathbf{3} \text { 746) }\end{array}$ & $\begin{array}{l}\text { SLNB after NACT, \% } \\
(\boldsymbol{n}=\mathbf{5 7 5})\end{array}$ \\
\hline IR & 98.0 & 97.0 \\
FNR & 4.0 & 9.0 \\
LRR & 0.9 & 1.2 \\
IR = identification rate; FNR = false-negative rate; LLR = local regional recurrence rate.
\end{tabular}

\section{Clinically node-positive patients prior to NACT}

The traditional approach has been that anyone with treatable breast cancer and a positive axillary node should have an ALND. This approach is undoubtedly correct if the patient remains clinically node-positive after NACT. Given the high rates of pCR following NACT, is ALND still necessary in patients who convert to nodenegative?

Restaging the axilla with ultrasound scanning, magnetic resonance imaging (MRI) or positron emission tomography (PET) remains inadequate in predicting pathological response; histology is then mandatory. The data from retrospective studies attempting SLNB in patients who converted to clinically node-negative after NACT showed unacceptably high FNRs. ${ }^{[1724,25]}$ However, the results from three multicentre prospective trials suggest that SLNB in this subgroup of patients may be acceptable.

The SENTINA trial ${ }^{[23]}$ divided 2234 patients who received NACT into four groups. One of these groups comprised clinically nodepositive patients who became clinically node-negative after NACT. They had an SLNB and completion ALND; the IR was $80.0 \%$ and the FNR $14.2 \%$, exceeding the acceptable FNR of $10.0 \%$. A very important finding was that the FNR reduced to $<10 \%$ if $\geq 3$ nodes were removed. The results also highlighted the benefit of a dual-tracer technique (patent blue and technetium-99m $\left({ }^{99 \mathrm{~m}} \mathrm{Tc}\right.$ )-nanocolloid) in this setting.

The American College of Surgeons Oncology Group (ACOSOG) Z-1071 trial investigated 649 patients who underwent SLNB and ALND after NACT, regardless of their clinical node status. ${ }^{[15]}$ The FNR was $12.6 \%$ but the FNR again decreased to $9.1 \%$, with dualtracer technique and removal of $\geq 3$ nodes. However, the trial was considered to have failed as the threshold of acceptable FNR $(<10 \%)$ was not achieved. A major shortcoming of the trial was the inclusion of patients without a complete clinical and radiological response.

In the Sentinel Node Biopsy Following NeoAdjuvant Chemotherapy in Biopsy Proven Node Positive Breast Cancer (SN-FNAC) trial, ${ }^{[26]}$ a dual tracer technique was used to locate sentinel lymph nodes in 153 patients who were node-negative after NACT. Metastases 


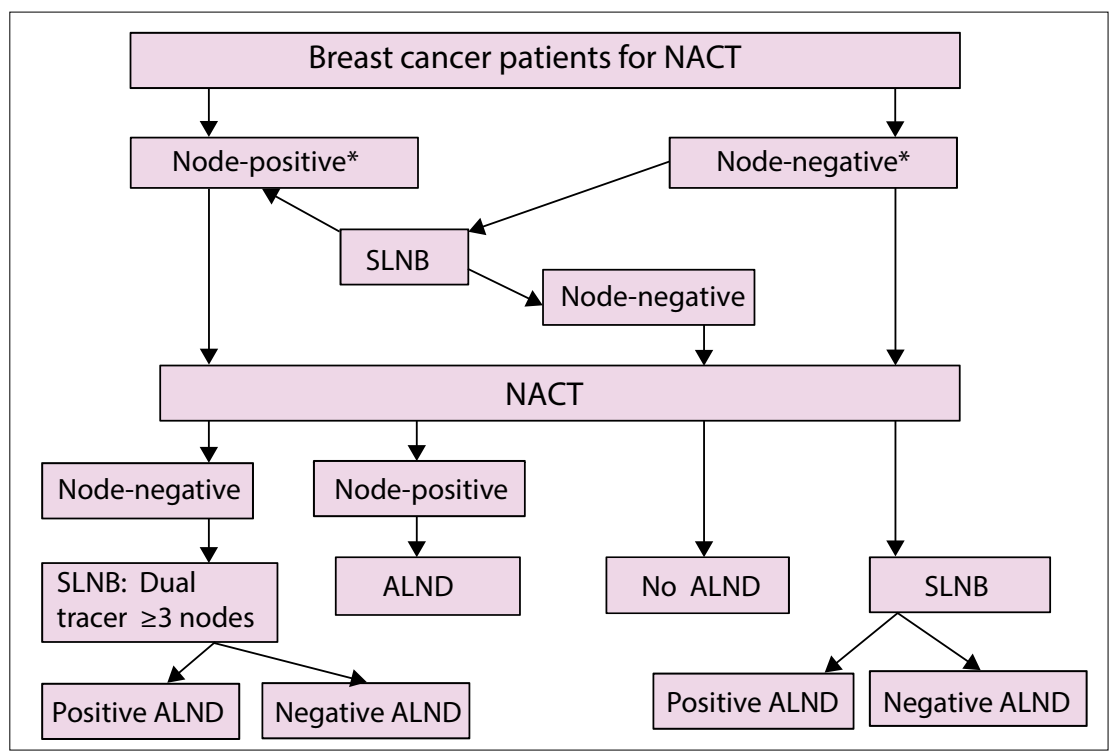

Fig. 1. Summary of recommendations with regard to the timing of SLNB when NACT is given. $\left({ }^{*}\right.$ Clinical and radiological node assessment and confirmed by fine-needle aspiration cytology.)

of any size were considered to be relevant and achieved an identification rate of $87.6 \%$. The FNR was $4.9 \%$ with $\geq 2$ sentinel nodes. In this trial the importance of using a dual tracer was emphasised. Use of immunohistochemistry to identify micrometastases and isolated tumour cells also reduced the FNR; the size of all metastases should be considered significant after NACT.

Although these trials pointed out the potential to reduce ALND in node-positive patients, there were still major concerns regarding the number of patients in whom this would be applicable. ${ }^{[27]}$ Importantly, the removed nodes must be true sentinel nodes, i.e. hot and/or blue nodes, and must not be randomly sampled. Mamtani et al. ${ }^{[16]}$ investigated patients with histologically proven node-positive disease who underwent NACT; $68.0 \%$ turned clinically node-negative and were eligible for SLNB. Of 128 SLNB attempts, $\geq 3$ sentinels were identified in $86.0 \%$, and $48.0 \%$ were histologically node-negative and spared an ALND. This study and a recent meta-analysis ${ }^{[28]}$ emphasise the major role of NACT to reduce ALND in patients with initial nodal disease and the feasibility of the procedure.

It must be pointed out that we lack longterm data on locoregional recurrence and survival in patients who had an SLNB only after converting from node-positive to nodenegative with NACT. Despite this, the current NCCN guidelines now allow for both ALND and SLNB in this patient group. ${ }^{[22]}$

In a further attempt to decrease the FNR, clipping of involved nodes to guide removal after NACT has been proposed. ${ }^{[29,30]}$ Given the increased cost and complexity of this approach, the clinical application in our setting is questionable.

In Fig. 1 the recommendations are summarised with regard to the timing of SLNB when NACT is given.

\section{Conclusion}

NACT has major potential to decrease the extent of surgery performed in the axilla.

If the nodal status is negative prior to starting NACT, it is safe to do an SLNB after NACT. If a node-positive patient has evidence of axillary disease after NACT, they should have an ALND. Patients who convert from node-positive to node-negative can have an SLNB, but:

- at least three nodes should be removed

- both patent blue and ${ }^{99 m} \mathrm{Tc}$-nanocolloid should be used to identify the nodes

- any size of lymph node metastasis should be considered to be node-positive and should prompt ALND.

\section{Acknowledgements. None.}

Author contributions. JE and SN were equally involved in the production of the article. Both have read the final manuscript.

\section{Funding. None.}

Conflicts of interest. None.

1. Veronesi U, Stafyla V. Grand challenges in surgica oncology. Front Oncol 2012;2:127. https://doi.org/10.3389/ oncology. Fron
fonc.2012.00127

2. Mauri D, Pavlidis N, Ioannidis JPA. Neoadjuvant versus adjuvant systemic treatment in breast cancer: A meta-analysis J Natl Cancer Inst 2005;97(3):188-194. https://doi.org/10.1093 jnci/dji021 chemotherapy on local-regional disease in women with operable breast cancer: Findings from National Surgical Adjuvant Breas and Bowel Project B-18. J Clin Oncol 1997;15(7):2483-2493.
3. Fisher B, Brown A, Mamounas E, et al. Effect of preoperative
4. Van Nes JGH, Putter H, Julien J-P, et al.; Cooperating Investigators of the EORTC. Preoperative chemotherapy is safe in early breas cancer, even after 10 years of follow-up; clinical and translational results from the EORTC trial 10902. Breast Cancer Res Trea 2009;115(1):101-113. https://doi.org/10.1007/s10549-008-0050-1

5. Von Minckwitz G. Preoperative therapy: What, when and for whom? Ann Oncol 2008;1(19)(Suppl 5):v113-v116. https://doi. org $/ 10.1093 /$ annonc/mdn323

6. Von Minckwitz G, Untch M, Nüesch E, et al. Impact of treatment characteristics on response of different breast cancer phenotypes: Pooled analysis of the German neo-adjuvant chemotherapy trials. Breast Cancer Res Treat 2011:125(1):145156. https://doi.org/10.1007/s10549-010-1228-x

156. https://doi.org/10.1007/s10549-010-1228-x
Cortazar P, Zhang L, Untch M, et al. Pathological complete 7. Cortazar P, Zhang L, Untch M, et al. Pathological complete
response and long-term clinical benefit in breast cancer: The CTNeoBC pooled analysis. Lancet 2014;384(9938):164-172. https://doi.org/10.1016/S0140-6736(13)62422-8

8. Fisher B. Laboratory and clinical research in breast cancer - a personal adventure: The David A Karnofsky Memorial Lecture. Cancer Res 1980;40(11):3863-3874

9. Fisher B, Anderson SJ. The breast cancer alternative hypothesis: Is there evidence to justify replacing it? J Clin Oncol 2010;28(3):366-374. https://doi.org/10.1200//CO .2009.26.8292

10. Cady B. Lymph node metastases: Indicators, but not governors of survival. Arch Surg 1984;119(9):1067-1072.

11. Mougalian SS, Hernandez M, Lei X, et al. Ten-year outcomes of patients with breast cancer with cytologically confirmed axillary lymph node metastases and pathologic complete response after primary systemic chemotherapy. JAMA Oncol 2016;2(4):508516. https://doi.org/10.1001/jamaoncol.2015.4935.

12. Koolen BB, Valdés Olmos RA, Wesseling J, et al. Early assessment of axillary response with 18F-FDG PET/CT during neoadjuvant chemotherapy in stage II - III breast cancer: Implications for surgical management of the axilla. Ann Surg Oncol 2013;20(7):2227-2235. management of the axilla. Ann Surg Oncol

13. Park S, Park JM, Cho JH, Park HS, Kim SI, Park B-W. Sentine lymph node biopsy after neoadjuvant chemotherapy in patients with cytologically proven node-positive breast cancer at diagnosis. Ann Surg Oncol 2013;20(9):2858-2865. https://doi. org/10.1245/s10434-013-2992-8

14. Hieken TJ, Boughey JC, Jones KN, Shah SS, Glazebrook KN. Imaging response and residual metastatic axillary lymph node disease after neoadjuvant chemotherapy for primary breast cancer. Ann Surg Oncol 2013;20(10):3199-3204. https://doi. cancer. Ann Surg Oncol 20132
org/10.1245/s10434-013-3118-z

15. Boughey JC, Suman VJ, Mittendorf EA, et al. Sentinel lymph node surgery after neoadjuvant chemotherapy in patients with nodepositive breast cancer: The ACOSOG Z1071 (Alliance) Clinical Trial. JAMA 2013;310(14):1455-1461. https://doi.org/10.1001/ jama.2013.278932

16. Mamtani A, Barrio AV, King TA, et al. How often does neoadjuvant chemotherapy avoid axillary dissection in patients with histologically confirmed nodal metastases? Results of with histogitly confred a prospective study. Ann Surg Oncol 2016;23(11):3467-3474

17. Alvarado R, Yi M, le-Petross $\mathrm{H}$, et al. The role for sentinel lymph node dissection after neoadjuvant chemotherapy in patients who present with node-positive breast cancer. Ann Surg Oncol 2012;19(10):31773184. https://doi.org/10.1245/s10434-012-2484-2

18. Dominici LS, Negron Gonzalez VM, Buzdar AU, et al. Cytologically proven axillary lymph node metastases are eradicated in patients receiving preoperative chemotherapy with concurrent trastuzumab for HER2-positive breast cancer. Cancer 2010:116(12):2884-2889 - htps://dois

9. Hunt KK, Yi M, Mittendorf EA, et al. Sentinel lymph node surgery after neoadjuvant chemotherapy is accurate and reduces
s. surgery after neoadjuvant chemotherapy is accurate and reduces
the need for axillary dissection in breast cancer patients. Trans the need for axillary dissection in breast cancer patients. Trans
Meet Am Surg Assoc 2009;127:189-197. https://doi.org/10.1097/ SLA.0b013e3181b8fd5e

20. Xing Y, Foy M, Cox DD, Kuerer HM, Hunt KK, Cormier JN. Meta-analysis of sentinel lymph node biopsy after preoperative chemotherapy in patients with breast cancer. Br J Sur 2006;93(5):539-546. https://doi.org/10.1002/bjs.5209

21. Van Deurzen CHM, Vriens BEPJ, Tjan-Heijnen VCG, et al Accuracy of sentinel node biopsy after neoadjuvant chemotherapy in breast cancer patients: A systematic review. Eur I Cancer 2009;45(18):3124-3130. https://doi.org/10.1016/j.ejca.2009.08.001 2009;45(18):3124-3130. https://doi.org/10.1016/j.ejca.2009.08.001 22. National Comprehensive Cancer Network. Breast Cancer (version
2.2016). https://www.nccn.org/store/login/login.aspx?ReturnURL=https://www.nccn.org/professionals/physician_gls/pdf/breast.pdf (accessed 2 May 2017)

23. Kuehn T, Bauerfeind I, Fehm T, et al. Sentinel-lymph-node biopsy in patients with breast cancer before and after neoadjuvan chemotherapy (SENTINA): A prospective, multicentre cohort study. Lancet Oncol 2013;14(7):609-618. https://doi.org/10.1016/ study. Lancet Oncol 2013:14

24. Shen J, Gilcrease MZ, Babiera GV, et al. Feasibility and accuracy of sentinel lymph node biopsy after preoperative chemotherapy in breast cancer patients with documented axillary metastases. Cancer 2007;109(7):1255-1263. https://doi.org/10.1002/cncr.22540

25. Takahashi M, Jinno H, Hayashida T, et al. Correlation between clinical nodal status and sentinel lymph node biopsy false negative rate after neoadjuvant chemotherapy. World J Sur 2012;36(12):2847-2852. https://doi.org/10.1007/s00268-012-1704-z 
26. Boileau J-F, Poirier B, Basik M, et al. Sentinel node biopsy after neoadjuvant chemotherapy in biopsyproven node-positive breast cancer: The SN FNAC study. J Clin Oncol 2015;33(3):258-264. https://doi. org/10.1200/JCO.2014.55.7827

27. King TA, Morrow M. Surgical issues in patients with breast cancer receiving neoadjuvant chemotherapy. Nat Rev Clin Oncol 2015;12(6):335-343, https://doi.org/10.1038/nrclinonc.2015.6 28. El Hage Chehade $\mathrm{H}$, Headon $\mathrm{H}$, El Tokhy $\mathrm{O}$, et al. Is sentinel lymph node biopsy a viable alternative to complete axillary dissection following neoadjuvant chemotherapy in women with node-positive breas cancer at diagnosis? An updated meta-analysis involving 3398 patients. Am J Surg 2016;212(5):969981. https://doi.org/10.1016/.j.amjsurg.2016.07.018
29. Caudle AS, Yang WT, Krishnamurthy S, et al. Improved axillary evaluation following neoadjuvant therapy for patients with node-positive breast cancer using selective evaluation of clipped nodes: Implementation of targeted axillary dissection. J Clin Oncol 2016;34(10):1072-1078. https://doi.org/10.1200/JCO.2015.64.009 30. Boughey JC, Ballman KV, le-Petross HT, et al. Identification and resection of clipped node decreases the false-negative rate of sentinel lymph node surgery in patients presenting with node-positive breast cancer (T0 - T4, N1 - N2) who receive neoadjuvant chemotherapy: Results from ACOSOG Z1071 (Alliance). Ann Surg 2016;263(4):802-807. https://doi.org/10.1097/SLA.0000000000001375

Accepted 6 February 2017. 